\title{
Les hypocrites de la protection de la jeunesse et leurs complices
}

\section{Rainer M. Kaelin}

Dr méd., spécialiste Médecine interne et Pneumologie, ancien vice-président de la ligue pulmonaire Suisse et vaudoise, vice-président d’OxyRomandie/ OxySuisse, membre FMH

Les cigarettiers tentent de convaincre le public et les parlementaires de leur conversion improbable: ils s'emploient désormais à ce que les fumeurs adultes passent à la cigarette électronique et aux produits alternatifs.

Lorsque le Dr Derek Yach, ancien médecin cadre de l'OMS, président désigné de la Fondation de Philip Morris (PM) «Pour un monde sans fumée», déclara que PM veut que la fumée cesse encore dans cette génération, il n'était pas crédible. Le supplément éditorial du 22 mai de la NZZ «Le retour n'est plus possible. PM cessera la production de cigarettes» suscitait également le scepticisme. Un nouvel élément de ces actions de relations publiques est l'interview du patron de British American Tobacco (BAT) «Personne n'est plus particulièrement fier de fumer» [1]. Le CEO de BAT, le non-fumeur Mads Larsen, est naturellement pour la protection de la jeunesse, il ne voudrait surtout pas que sa «fille de douze ans rentre à la maison et lui dise qu'elle a commencé à vapoter». Au sujet du marketing agressif pour les cigarettes électroniques «Juul», les parents d'adolescents seront d'accord avec lui lorsqu'il affirme: «Ce qui se passe aux Etat-Unis est choquant! Nous devons faire en sorte que la Suisse ne subisse pas une évolution pareille.» Tout comme Jonathan Green de Juul Labs [2], il déclare: «Je mets ma

\section{Non-fumeur, le CEO de British American}

Tobacco est évidemment pour la protection de la jeunesse, affirme-t-il dans une interview.

main au feu que nous vendons uniquement à des personnes de 18 ans ou plus, donc à des fumeurs adultes. C'est ce que prévoit notre autocontrôle qui existe depuis 2005.»

\section{Affirmations contradictoires}

Que BAT, comme Juul et PM, veut vendre les nouveaux produits uniquement aux fumeurs adultes, ne peut pas être une intention honnête. Car sans le recrute- ment de nouveaux dépendants de la nicotine, ce modèle commercial verra son marché s'assécher d'ici une génération. L'offre de cigarettes électroniques doit donc séduire la
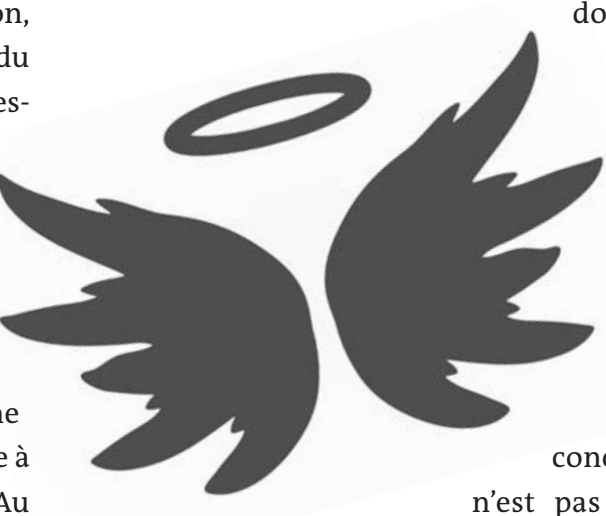
génération de nouveaux fumeurs, soit les jeunes. Et en effet, Larsen veut «gagner des parts de marché de nos concurrents. Ceci n'est pas possible sans publicité». Le soi-disant autocontrôle de la publicité, censé empêcher la consommation de tabac parmi les jeunes, est une farce: Swiss Cigarette (c.-à-d. PM, BAT et Japan Tobacco international, JTI) s'est accordé en 2005 avec la «commission de loyauté» (CL) pour que celle-ci se charge de juger les infractions au code d'honneur. Contrairement aux plaintes d'autres domaines, le règlement de la CL prévoit que les infractions commises par l'industrie du tabac ne seraient pas sanctionnées, mais transmises en tant que recommandations à ... Swiss Cigarette [3]! Rien d'étonnant donc que Larsen refuse l'initiative populaire "Oui pour la protection des enfants et des jeunes contre la publicité pour le tabac». Car "presque la moitié des dépenses publicitaires de BAT vont actuellement vers les nouveaux produits. Par ailleurs: Quid de l'alcool, du gras et du sucre, qui peuvent également nuire à la santé? En toute conséquence, la publicité pour ces aliments devrait également être interdite.» Utilisé par l'USAM, les publicitaires et l'UDC [4], ce vocabulaire est désormais monnaie courante depuis le 
siècle dernier. La preuve dans un document interne de R. Reynolds Tobacco (racheté par JTI) datant de 1993, qui taisait que la nocivité du tabac provenait du grand pouvoir addictif de la nicotine auprès des jeunes [5]. L'ambivalence du citoyen responsable Larsen et du CEO de BAT apparaît lorsqu'il commente l'affirmation classique de sa branche selon laquelle le tabac serait un produit de plaisir comme un autre: «La nicotine a un pouvoir addictif élevé, c'est pourquoi une consommation modérée - contrairement au verre de rouge - est problématique à moyen et à long terme.» Il entonne

\section{Depuis des années, les chiffres officiels sous- estiment largement la prévalence des fumeurs en Suisse. Mais on se garde bien de le dire.}

néanmoins le refrain connu: «L'Etat ne peut influencer le comportement des consommateurs que de manière limitée. En France, notre branche n'est pas du tout autorisée à faire de la publicité, et pourtant, là-bas, beaucoup plus de personnes fument qu'en Suisse.» Mais il tait le fait que les chiffres officiels sous-estiment, depuis longtemps et de manière flagrante, la prévalence des fumeurs en Suisse [6].

\section{La législation libérale suisse du tabac}

Dans la même édition de la NZZ, on trouve l'article «La législation suisse relativement libérale du tabac devient plus stricte. Sept réponses concernant le changement du marché du tabac». Les auteurs mentionnent l'interdiction de fumer dans les gares, l'initiative populaire «Oui à la protection des enfants et des jeunes contre la publicité pour le tabac» et les réglementations débattues de la loi sur les produits du tabac (LPtab): l'interdiction de la vente aux mineurs, les achats tests pour l'imposer et la prohibition de la publicité visant spécialement les jeunes. Ce qui serait théoriquement déjà le cas, si l'autorégulation de la branche par la CL était respectée. Concernant les cigarettes électroniques et le snus, on parle de règles "différenciées» et que la publicité, le marketing et le sponsoring pour ces produits devraient être réglés de la même manière que pour les produits conventionnels, c'est-à-dire quasiment pas du tout, puisque cela ne porterait que sur la publicité conventionnelle et non pas sur les méthodes modernes de promotion commerciale. Il semble qu'on ait accédé aux demandes de l'industrie pour lui accorder des exceptions: les produits "alternatifs» seraient réglés dans une catégorie à part, ils devraient porter une mise en garde générale, mais sans les images épouvantables des paquets de cigarettes. L'article factuel, mais pas du tout critique, omet de mentionner la sous-estimation des quotas des fumeurs en Suisse [6], et ne commente pas la convention cadre de la lutte contre le tabagisme (CCLAT) qui attend sa ratification parlementaire depuis 2004. La CCLAT préconise que la publicité, la promotion et le sponsoring soient «globalement" interdits et les mesures de prévention des Etats protégées contre leur neutralisation par l'industrie. Le lecteur de la NZZ n'apprend pas que précisément le conseiller aux Etats uranais Josef Dittli demanda dans la séance du la commission de la santé du 19 février que la LPTab soit complétée par des interdictions de la publicité pour permettre la ratification de la CCLAT (7). En 2016, le même politicien avait déclenché le retour de la LPTab au Conseil fédéral, car il affirmait ne pas connaître de chiffres prouvant que les interdictions de la publicité feraient baisser le taux de fumeurs!

\section{Le conflit irréconciliable}

Que l'industrie du tabac tente par tous les moyens de convaincre les parlementaires et l'opinion publique que la nicotine serait banale et que seulement le tabac brûlé serait responsable de l'épidémie tabagique n'est pas étonnant. Car reconnaître que c'est la dépendance de la nicotine ancrée dans les cerveaux des jeunes qui cause l'épidémie du tabac et ses conséquences remettrait fondamentalement en question son modèle d'affaires. Et montrerait l'industrie comme ennemi implacable de la santé publique. Mais ce fait est également le fondement des exigences de la CCLAT qui se base sur l'évidence scientifique, en particulier que l'interdiction de la publicité, de la promotion et du sponsoring doit être globale pour garantir une protection de la jeunesse. L'argumentation fallacieuse «diminution du risque par des produits moins nocifs» était déjà la base du marketing des cigarettes à filtre et des "légères», lorsqu'on commença à mesurer la nicotine et les composantes carcinogènes de la fumée. Par cette astuce,

Il n'est pas étonnant que l'industrie du tabac tente par tous les moyens de convaincre politiciens et citoyens que la nicotine est banale.

tout comme plus tard l'industrie automobile, les cigarettiers trompèrent le public et les autorités. Car de ces produits conventionnels il n'a jamais été prouvé qu' ils seraient moins nocifs; ils ont en revanche sensiblement augmenté la consommation chez les jeunes et les femmes. Depuis l'apparition de l'e-cigarette, les publireportages sur la "réduction des risques» et la banalisation de la nicotine se trouvent à nouveau fréquemment parmi les contenus payés des médias. Les 
messages répandus, guère questionnés par ces articles, sont par exemple la «moindre nocivité de 95\%» qui fait l'amalgame entre le contenu en toxiques et le risque (Prof. Etter 2013), «La publicité, c'est l'information du client adulte», «Pour un produit légal, on peut faire de la publicité légale» (Filippo Lombardi, conseiller aux Etats, président de Communication Suisse, 2016), «Changement de cap dans la politique de l'addiction en Suisse» (Fachverband Sucht 2017), "Alternatives raisonnables aux cigarettes de tabac» (Prof. Kunze, 2017).

\section{Intoxication de l'opinion publique et du processus démocratique}

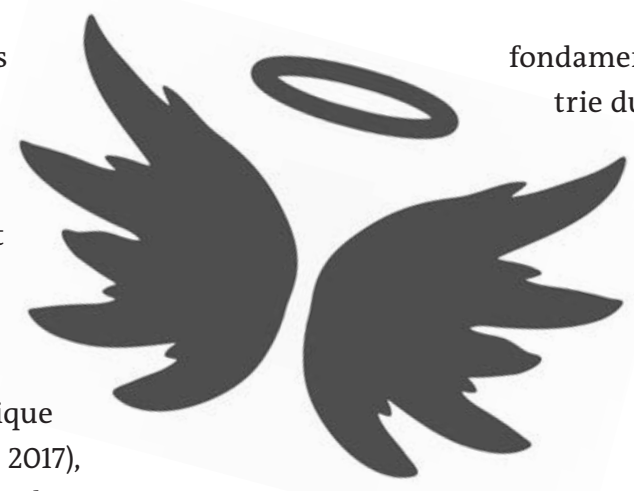

tion cadre de l'OMS, ou sur l'initiative populaire «Oui à la protection des enfants et des jeunes contre la publicité du tabac».

Références

1 NZZ vom 1.6.2019: «Niemand ist mehr besonders stolz darauf zu rauchen". Interview mit Mads Larsen, von Erich Aschwanden und Simon Hehli.

2 Tages-Anzeiger vom 5.12.2018: «Ich bin selber Vater von zwei Kindern".

3 Kaelin RM. Protection de la jeunesse sans interdiction de la publicité? Bull Med Suisses. 2017;98(41):1347-49.

4 Loi fédérale sur les produits du tabac (LPTab): réponse à la consultation de l'UDC. 9.9.2014.

5 R. J. Reynolds Tobacco International: Swiss Advertising Ban Case Study. 1993 November 28. RJ Reynolds Records (Author unknown). Internet: https://www.industrydocuments.ucsf.edu/docs/ pzmh0077 (accessed 22.5.2019).

6 J. Jacob, J. Cornuz, P. Diethelm. Prevalence of Tobacco smoking in Switzerland: do reported numbers underestimate reality? Swiss Medical Weekly. 2017;147:w14437.

7 Lorenz Hongegger. Lukratives Mandat: Der «Briefträger der Tabaklobby» wechselt die Seite. Schweiz am Wochenende, 2.3.2019. mesures publicitaires et promotionnelles, ainsi qu'un large trafic d'influence digne des cartels. Ces manipulations (et non pas la plante de tabac) sont responsables du problème de santé publique qu'est l'épidémie mondiale du tabagisme. Parlementaires et professionnels des médias seraient bien inspirés de tirer du conflit

elles ont réussi à camoufler à la société et aux autorités ces manipulations et leurs séquelles multiples par des

Crédit photo
(c) Annepunch

() Annepunch | Dreamstime.com
Correspondance:

Plantay 53

CH-1163 Etoy VD

palmier.kaelin[at]

bluewin.ch 